\title{
Experimental investigation to characterize simple versus multi scaling analysis of hydraulic conductivity at a mesoscale
}

\author{
Guglielmo Federico Antonio Brunetti ${ }^{1}$ Samuele De Bartolo ${ }^{2}$ Carmine Fallico ${ }^{1} \cdot$ Ferdinando Frega $^{1}$. \\ Maria Fernanda Rivera Velásquez ${ }^{3} \cdot$ Gerardo Severino $^{4}$ (10
}

Accepted: 12 August 2021 / Published online: 21 August 2021

(c) The Author(s) 2021

\begin{abstract}
The spatial variability of the aquifers' hydraulic properties can be satisfactorily described by means of scaling laws. The latter enable one to relate the small (typically laboratory) scale to the larger (typically formation/regional) ones, therefore leading de facto to an upscaling procedure. In the present study, we are concerned with the spatial variability of the hydraulic conductivity $K$ into a strongly heterogeneous porous formation. A strategy, allowing one to identify correctly the single/multiple scaling of $K$, is applied for the first time to a large caisson, where the medium was packed. In particular, we show how to identify the various scaling ranges with special emphasis on the determination of the related cut-off limits. Finally, we illustrate how the heterogeneity enhances with the increasing scale of observation, by identifying the proper law accounting for the transition from the laboratory to the field scale. Results of the present study are of paramount utility for the proper design of pumping tests in formations where the degree of spatial variability of the hydraulic conductivity does not allow regarding them as "weakly heterogeneous", as well as for the study of dispersion mechanisms.
\end{abstract}

Keywords Scaling behavior $\cdot$ Hydraulic conductivity $\cdot$ Slug test $\cdot$ Power law

\section{Introduction}

The knowledge of the hydraulic conductivity is crucial to model flow and transport through porous media. The hydraulic conductivity changes over several orders of magnitude (Harp and Vesselinov 2010), depending upon the scale (support) under consideration (Di Federico and Neuman 1998; Di Federico et al. 1999; Neuman and Di Federico 2003). We are interested in the scaling behavior of the hydraulic conductivity, which has been assessed in the past (Fallico et al. 2010, 2012, 2016; Fallico 2014;

Gerardo Severino

severino@unina.it

1 Department of Civil Engineering, University of Calabria, 87036 Rende, Cosenza, Italy

2 Department of Innovation Engineering, University of Salento, 73100 Lecce, Italy

3 Escuela Superior Politécnica de Chimborazo, Riobamba 060155, Ecuador

4 Department of Agricultural Sciences, University of Naples Federico II, 80055 Portici, Naples, Italy
Severino and Santini 2005; Severino et al. 2009; Severino and Coppola 2012), by highlighting an increase of the $k$ values with increasing support (Fallico et al. 2020; Hunt 2006; Sánchez-Vila et al. 1996). Within such a view, a fundamental role is played by the heterogeneity of the porous formation. In fact, at smaller (laboratory) scales, the heterogeneity is mainly related to the pore sizes and their shape, whereas at larger (field) scales, it is related to the connectivity and tortuosity of pores (Bird and Perrier 2010; Bouma 1982; Giménez et al. 1999; Knudby and Carrera 2006; Severino 2011a; Yanuka et al. 1986). Between these two scales, a scaling (intermediate) range can be identified, which generally covers a length scale ranging from 0.5 to $10 \mathrm{~m}$.

The spatial distribution of the hydraulic conductivity is characterized by means of geostatistics (Severino et al. 2019). Alternatively, one can employ a scaling law relating the large scale behavior to the local scale(s). Such an approach facilitates the study of particular (i.e. local) phenomena, which often influence flow and transport at the larger (macro) scales (Indelman 2004; Severino 2011b; Severino et al. 2011; Severino and De Bartolo 2015; 
Broyda et al. 2010). This highlights the importance of the intermediate scale (Severino et al. 2010; Fallico et al. 2018).

In the present paper, we aim at verifying the scaling behavior of the hydraulic conductivity at the mesoscale. Like any characteristic length scale (an extensive exposition can be found in Dagan 1989), even the mesoscale expresses the average distance over which the conductivity is correlated, although the uncertainty has still a minor impact on flow (a real world example can be found in Fallico et al. 2018). This is done by means of a massive experimental campaign based on slug tests (Fallico et al. 2016) in an artificially packed, largely heterogeneous porous formation. Then, we move on checking whether the modalities of the $K$-scaling are similar to those that are generally detected into mildly heterogeneous formations. Hence, a discussion to highlight common vs different grounds with the past literature is carried out, and we end up with a few concluding remarks.

\section{Material and methods}

\subsection{Experimental laboratory set-up and $K$ measurements by slug tests}

To carry out the flow experiments, a confined aquifer, equipped with 10 fully penetrating wells, was packed. The aquifer was assembled within a steel box, with a square base ( $2 \mathrm{~m}$ side) and $1 \mathrm{~m}$ height. Inside the box, at a distance of $5 \mathrm{~cm}$ from the walls of the box, and along the whole perimeter, vertical metallic supports were fixed, to which a metal net was anchored, and covered by a geotextile to prevent loss of the porous material. This configuration allowed to contain the porous medium, within the metal mesh, so as to constitute between this and the box walls a space useful to keep fixed the hydraulic heads. Ten piezometers, placed approximately according to a spiral, with a distance $(d)$ gradually increasing from the center were inserted. Each piezometer was located at $10 \mathrm{~cm}$ away from the previous, along directions increased by $45^{\circ}$-step from the previous one, except for the piezometer No. 10, placed to reduce the proximity to the edge. Each piezometer, with inner radius of $1,4 \mathrm{~cm}$, was screened to result fully penetrating. To avoid intrusions of soil particles within them, the piezometers were coated with the same geotextile placed on the metal mesh. To set with enough precision the water level inside the box and, therefore, the aquifer hydraulic head, on two opposite walls of the box, two small PVC containers, with inside a rectangular weir, height-adjustable and hydraulically connected to the perimetric head chamber, were realized. The porous medium was subjected to careful particle size analysis (silt: $1.20 \%$; sand: $86.79 \%$; gravel: $12.01 \%$; effective diameter $d_{10}=0.19 \mathrm{~mm}$ and uniformity coefficient $U=d_{60} /$ $d_{10}=5.16$ ). To realize the roof of the confined aquifer, PVC panels of $2 \mathrm{~mm}$ of thickness were used. The thickness $B=25 \mathrm{~cm}$ of the aquifer was realized by successive layers, each subjected to some wetting/drying cycles, to favour compaction. Ten pressure transducers, placed at the bottom of each well, were used. The experimental apparatus was tested several times before carrying out the flow experiments (Fallico et al. 2018). In Fig. 1 the layout of the wells is shown, with a transverse representation of the metal box, and a picture of the whole apparatus.

Several slug tests were performed. An initial undisturbed hydraulic head of approximately $0.4 \mathrm{~m}$ was used for all the experiments. This condition caused the aquifer to be slightly under pressure. In particular, six slug tests were executed by injecting an increasing water volume, $V=30$, 40, 60, 70, 80 and $90 \mathrm{ml}$ in the central well. These volumes were calibrated according to water quantities which were acceptable as a function of the dimensions of the laboratory set-up without causing undesired overflow and ensuring that the perturbed hydraulic head in the aquifer did not interact with the solid walls surrounding the experimental aquifer. The occurrence of the above mentioned flow conditions was checked for each test through the monitoring of the hydraulic head in the injection and observation wells. Specifically, the monitoring of hydraulic heads in the observation wells was also performed to verify the suitability of the boundary conditions. Moreover, attention was also paid to check the reaching of the initial undisturbed conditions between successive tests.

\subsection{Methods}

\subsubsection{Analysis of head data and $\mathrm{k}$ values determination}

The $K$ values were determined by the method of Hvorslev (1951). This method assumes the specific storage to be so small that its effects can be neglected, i.e. quasi-steadystate conditions. Moreover, for this method the slug volume can not necessarily be introduced in an instantaneous manner and, at last, lateral constant-head boundaries are applied at a finite distance $\left(R_{e}\right)$. Under these assumptions, the analytical solution of the flow equation write as:

$\ln \left(\frac{H(t)}{H_{0}}\right)=-\frac{2 K B t}{r_{c}^{2} \ln \left(R_{e} / r_{w}\right)}$

where $K$ is the horizontal hydraulic conductivity $\left[\mathrm{LT}^{-1}\right.$ ], $H(t)$ is the displacement of hydraulic head in well from static conditions [L], $H_{O}$ is the initial hydraulic head [L], $r_{c}$ is the effective radius of well casing [L], $r_{w}$ is effective radius of well screen [L], $R_{e}$ is the effective radius parameter [L] and $B$ was already defined above (Hvorslev 
(a)

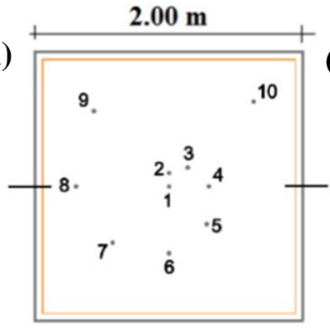

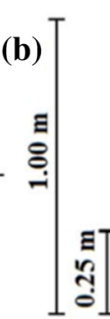

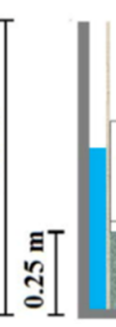

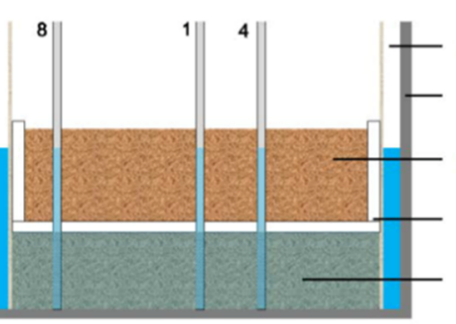

Hydraulic head space

Metallic box

Sandy covering layer

Waterproof PVC laye

Confined aquifer (c)

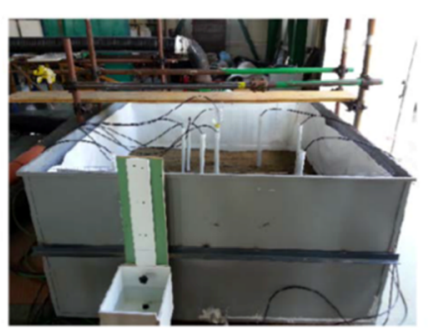

Fig. 1 a Planimetric layout of the piezometers; $\mathbf{b}$ stratigraphic layout; $\mathbf{c}$ experimental apparatus

1951; Butler 1997). In any case, before determining the $K$ values, the data series of hydraulic head detected at the well and at each piezometer were subjected to a careful smoothing analysis by using wavelet transform (Aristodemo et al. 2018).

\subsubsection{Analysis of scaling behavior}

The dependence of hydraulic conductivity upon the measurement support (Fallico et al. 2012, 2016; SchulzeMakuch and Cherkauer 1998) was experimentally verified by slug tests performed in the above described confined aquifer. It is worth reminding here that, $K$-measurements depend upon:

$s=R-d$

where $R$ is the radius of influence, measured for each injection volume, and $d$ is the distance of each piezometer from the injection well. To describe the scaling behavior of $K$, a simple power type law was used, i.e.

$P=a x^{b}$

where $P$ is the parameter examined (in this case the hydraulic conductivity [LT $\left.{ }^{-1}\right]$ ), $x$ is the scale parameter (as distances [L]), $a$ is a parameter related to the structure and heterogeneity of the medium, and $b[-]$ is the scaling index (or crowding index), which takes into account the type of flow in the porous medium and the actual dimensions of the measurement scale (Schulze-Makuch and Cherkauer 1998).

Overall, a power law is justified if the signal of the hydrological process at the stake is bounded by cut-offs. To determine the cut-off limits, a simple fitting procedure has been used (Fallico et al. 2016, 2018; De Bartolo et al. 2013). In addition, the methodology mentioned above is useful to highlight the possible dependence on more than one scale (Fallico et al. 2016). In this case the power law is slightly generalized as follows:

$\tilde{P}(x)=\sum_{i=1}^{N} a_{i} x^{b_{i}}$ where the integer $i$ indicates the $i$-th partial scaling range $(N>1)$, and the other terms were already defined above. The relation (4) can be regarded as the overall overlapping effects, each one described by the Eq. (3).

\section{Results and discussion}

The $K$ values measured for each injected volume, and the relative values of the radius of influence, are shown in Table 1.

Table 1 shows that the $K$ values are of the same order of magnitude. The $K$ values, both at the injection well and at the piezometers, are shown in Table 2 along with the scale parameter (Eq. (2)).

It is seen that the heads detected in the central injection well are the highest, and the $K$ values determined therein are representative of the confined aquifer volume identifiable with a cylinder of radius equal to the radius of influence and height equal to the thickness of the aquifer. For each of these data sets, a careful statistical analysis was carried out (Table 3).

It is seen that the mean value varies, depending on the volume of injection, between $1.37 \times 10^{-4} \mathrm{~m} / \mathrm{s}$ and $2.98 \times 10^{-4} \mathrm{~m} / \mathrm{s}$. The maximum values of the variance (VAR), standard deviation (SD), standard error (SE) and coefficient of variation (VC) are found for an injection volume of $0.08 \mathrm{~L}$, while the corresponding minimum values are found for an injection volume of $0.04 \mathrm{~L}$. Only two Kurtosis values, relative to volumes $0.03 \mathrm{~L}$ and $0.04 \mathrm{~L}$, are positive, while the other four are negative, showing a trend towards a flattening of the distribution, compared to the normal one. Skewness values are all positive, highlighting an asymmetric trend. Inspection from Table 2 shows a spatial variability of K with s. Such a dependence has been analyzed by means of a simple power law (3), by identifying the pair $(a, b)$ for each injected volume (Table 4).

The resulting power laws are depicted, for each injected volume and together with the measured $\mathrm{K}$-value, in the Fig. 2.

The most interesting feature that is detected from Fig. 2 is a change in the slope with increasing $s$. This can be better 
Table 1 Values of $K$ as function of the injected volume $\mathrm{V}$, and the radius of influence $R$

\begin{tabular}{lll}
\hline Injected volumes $V(\mathrm{~L})$ & Radii of influence measured $R(\mathrm{~m})$ & $K(\mathrm{~m} / \mathrm{s})$ \\
\hline 0.03 & 0.592 & $2.34 \times 10^{-4}$ \\
0.04 & 0.707 & $2.36 \times 10^{-4}$ \\
0.06 & 0.797 & $2.58 \times 10^{-4}$ \\
0.07 & 0.846 & $3.30 \times 10^{-4}$ \\
0.08 & 0.868 & $4.16 \times 10^{-4}$ \\
0.09 & 0.943 & $4.48 \times 10^{-4}$ \\
\hline
\end{tabular}

Table 2 Values of $K$ for each injection volume and their relative $s$-values

\begin{tabular}{|c|c|c|c|c|c|c|c|c|c|c|c|}
\hline \multicolumn{2}{|c|}{$V=0.03 \mathrm{~L}$} & \multicolumn{2}{|c|}{$V=0.04 \mathrm{~L}$} & \multicolumn{2}{|c|}{$V=0.06 \mathrm{~L}$} & \multicolumn{2}{|c|}{$V=0.07 \mathrm{~L}$} & \multicolumn{2}{|c|}{$V=0.08 \mathrm{~L}$} & \multicolumn{2}{|c|}{$V=0.09 \mathrm{~L}$} \\
\hline$s(\mathrm{~m})$ & $K(\mathrm{~m} / \mathrm{s})$ & $s(\mathrm{~m})$ & $K(\mathrm{~m} / \mathrm{s})$ & $s(\mathrm{~m})$ & $K(\mathrm{~m} / \mathrm{s})$ & $s(\mathrm{~m})$ & $K(\mathrm{~m} / \mathrm{s})$ & $s(\mathrm{~m})$ & $K(\mathrm{~m} / \mathrm{s})$ & $s(\mathrm{~m})$ & $K(\mathrm{~m} / \mathrm{s})$ \\
\hline 0.092 & $9.38 \times 10^{-5}$ & 0.007 & $9.90 \times 10^{-5}$ & 0.097 & $1.04 \times 10^{-4}$ & 0.046 & $1.17 \times 10^{-4}$ & 0.068 & $1.21 \times 10^{-4}$ & 0.043 & $1.49 \times 10^{-4}$ \\
\hline 0.192 & $9.57 \times 10^{-5}$ & 0.107 & $1.02 \times 10^{-4}$ & 0.197 & $1.11 \times 10^{-4}$ & 0.146 & $1.21 \times 10^{-4}$ & 0.168 & $1.35 \times 10^{-4}$ & 0.143 & $1.63 \times 10^{-4}$ \\
\hline 0.292 & $1.27 \times 10^{-4}$ & 0.207 & $1.29 \times 10^{-4}$ & 0.297 & $1.34 \times 10^{-4}$ & 0.246 & $1.40 \times 10^{-4}$ & 0.268 & $1.48 \times 10^{-4}$ & 0.243 & $1.72 \times 10^{-4}$ \\
\hline 0.392 & $1.32 \times 10^{-4}$ & 0.307 & $1.35 \times 10^{-4}$ & 0.397 & $1.40 \times 10^{-4}$ & 0.346 & $1.52 \times 10^{-4}$ & 0.368 & $1.61 \times 10^{-4}$ & 0.343 & $2.17 \times 10^{-4}$ \\
\hline 0.492 & $1.41 \times 10^{-4}$ & 0.407 & $1.47 \times 10^{-4}$ & 0.497 & $1.45 \times 10^{-4}$ & 0.446 & $1.90 \times 10^{-4}$ & 0.468 & $2.33 \times 10^{-4}$ & 0.443 & $2.51 \times 10^{-4}$ \\
\hline 0.592 & $2.34 \times 10^{-4}$ & 0.507 & $1.52 \times 10^{-4}$ & 0.597 & $1.79 \times 10^{-4}$ & 0.546 & $2.38 \times 10^{-4}$ & 0.568 & $3.42 \times 10^{-4}$ & 0.543 & $3.33 \times 10^{-4}$ \\
\hline- & - & 0.607 & $1.80 \times 10^{-4}$ & 0.697 & $2.24 \times 10^{-4}$ & 0.646 & $3.08 \times 10^{-4}$ & 0.668 & $3.74 \times 10^{-4}$ & 0.643 & $3.95 \times 10^{-4}$ \\
\hline- & - & 0.707 & $2.36 \times 10^{-4}$ & 0.797 & $2.58 \times 10^{-4}$ & 0.746 & $3.17 \times 10^{-4}$ & 0.768 & $3.93 \times 10^{-4}$ & 0.743 & $4.15 \times 10^{-4}$ \\
\hline- & - & - & - & - & - & 0.846 & $3.30 \times 10^{-4}$ & 0.868 & $4.16 \times 10^{-4}$ & 0.843 & $4.36 \times 10^{-4}$ \\
\hline- & - & - & - & - & - & - & - & - & - & 0.943 & $4.48 \times 10^{-4}$ \\
\hline
\end{tabular}

Table 3 Main statistical parameters of the $K$ values determined in the injection well and in the piezometers

\begin{tabular}{|c|c|c|c|c|c|c|}
\hline \multirow[t]{2}{*}{ Statistical parameters } & \multicolumn{6}{|c|}{ global data sets for each injection volume } \\
\hline & $0.03 \mathrm{~L}$ & $0.04 \mathrm{~L}$ & $0.06 \mathrm{~L}$ & $0.07 \mathrm{~L}$ & $0.08 \mathrm{~L}$ & $0.09 \mathrm{~L}$ \\
\hline $\mathrm{N}$ & 6 & 8 & 8 & 9 & 9 & 10 \\
\hline $\min$ & $9.38 \times 10^{-5}$ & $9.90 \times 10^{-5}$ & $1.04 \times 10^{-4}$ & $1.17 \times 10^{-4}$ & $1.21 \times 10^{-4}$ & $1.49 \times 10^{-4}$ \\
\hline $\max$ & $2.34 \times 10^{-4}$ & $2.36 \times 10^{-4}$ & $2.58 \times 10^{-4}$ & $3.30 \times 10^{-4}$ & $4.16 \times 10^{-4}$ & $4.48 \times 10^{-4}$ \\
\hline mean & $1.37 \times 10^{-4}$ & $1.48 \times 10^{-4}$ & $1.62 \times 10^{-4}$ & $2.13 \times 10^{-4}$ & $2.58 \times 10^{-4}$ & $2.98 \times 10^{-4}$ \\
\hline VAR & $2.62 \times 10^{-9}$ & $1.98 \times 10^{-9}$ & $2.98 \times 10^{-9}$ & $7.68 \times 10^{-9}$ & $1.50 \times 10^{-8}$ & $1.45 \times 10^{-8}$ \\
\hline SD & $5.12 \times 10^{-5}$ & $4.45 \times 10^{-5}$ & $5.46 \times 10^{-5}$ & $8.76 \times 10^{-5}$ & $1.22 \times 10^{-4}$ & $1.21 \times 10^{-4}$ \\
\hline SE & $2.09 \times 10^{-5}$ & $1.57 \times 10^{-5}$ & $1.93 \times 10^{-5}$ & $2.92 \times 10^{-5}$ & $4.08 \times 10^{-5}$ & $3.81 \times 10^{-5}$ \\
\hline $\mathrm{VC}$ & $3.73 \times 10^{-1}$ & $3.01 \times 10^{-1}$ & $3.37 \times 10^{-1}$ & $4.12 \times 10^{-1}$ & $4.74 \times 10^{-1}$ & $4.05 \times 10^{-1}$ \\
\hline Kurtosis & 3.32219 & 1.44701 & -0.303000 & -1.90004 & -2.17760 & -1.99717 \\
\hline Skewness & 1.67520 & 1.10593 & 0.90150 & 0.33209 & 0.16035 & 0.01548 \\
\hline
\end{tabular}

visualized in the Fig. 3, which also suggests that the best power law is presumably a combination of two models of the type (3), i.e.

$\begin{array}{lll}k=a_{1} s^{b_{1}} & \text { for } & s_{I, \text { low }}<s<s_{I, \text { upp }} \\ k=a_{2} s^{b_{2}} & \text { for } & s_{I I, \text { low }}<s<s_{I I, \text { upp }}\end{array}$ where $a_{1}$ and $b_{1}$ are the parameters of the Eq. (3) relative to the I range and similarly for $a_{2}$ and $b_{2}$, relative to the II range.

For each of the data sets related to the injection volumes considered and for both I and II ranges, the values of the lower and upper cut-off limits are shown in Table 5, while the scaling laws were also determined, and the 
corresponding values of the parameters $a$ and $b$ and $\mathrm{R}^{2}$ are shown in Table 6.

Table 4 Parameters $a$ and $b$ of the scaling laws, determined by Eq. (3) along with the corresponding values of the determination coefficient $\mathrm{R}^{2}$

\begin{tabular}{llll}
\hline Volumes injected (L) & $a\left(\mathrm{~m}^{(1-b)} / \mathrm{s}\right)$ & $b$ & $\mathrm{R}^{2}$ \\
\hline 0.03 & $2.17 \times 10^{-4}$ & 0.409 & 0.715 \\
0.04 & $2.21 \times 10^{-4}$ & 0.366 & 0.842 \\
0.06 & $2.30 \times 10^{-4}$ & 0.407 & 0.812 \\
0.07 & $3.03 \times 10^{-4}$ & 0.397 & 0.773 \\
0.08 & $4.00 \times 10^{-4}$ & 0.553 & 0.811 \\
0.09 & $4.15 \times 10^{-4}$ & 0.414 & 0.820 \\
\hline
\end{tabular}

It is seen from Table 6 , that the values of the coefficient $a_{1}$ are all very similar, varying between $1.66 \times 10^{-4} \mathrm{~m}^{(1-\mathrm{b})} / \mathrm{s}$ and $1.92 \times 10^{-4} \mathrm{~m}^{(1-\mathrm{b})} / \mathrm{s}$, while the values of $b_{1}$, also very close, are variable between 0.081 and 0.266. Similarly for the II range, the values of $a_{2}$ are very close, varying between $3.45 \times 10^{-4} \mathrm{~m}^{(1-b)} / \mathrm{s}$ and $9.83 \times 10^{-4} \mathrm{~m}^{(1-\mathrm{b})} / \mathrm{s}$, while the values of $b_{2}$, also very similar, vary between 0.765 and 2.738 . In particular, the values of $a_{2}$ are significantly higher than the corresponding values of $a_{1}$, although both have the same order of magnitude. The values of the $\mathrm{R}^{2}$ are always close to 1 . Figure 4 shows the scaling laws related to the I and II ranges.

As expected, it is seen that, for all the injection volumes considered, the trends of the straight lines representing the scaling laws related to the II range have a slope greater than the corresponding ones of the I range.

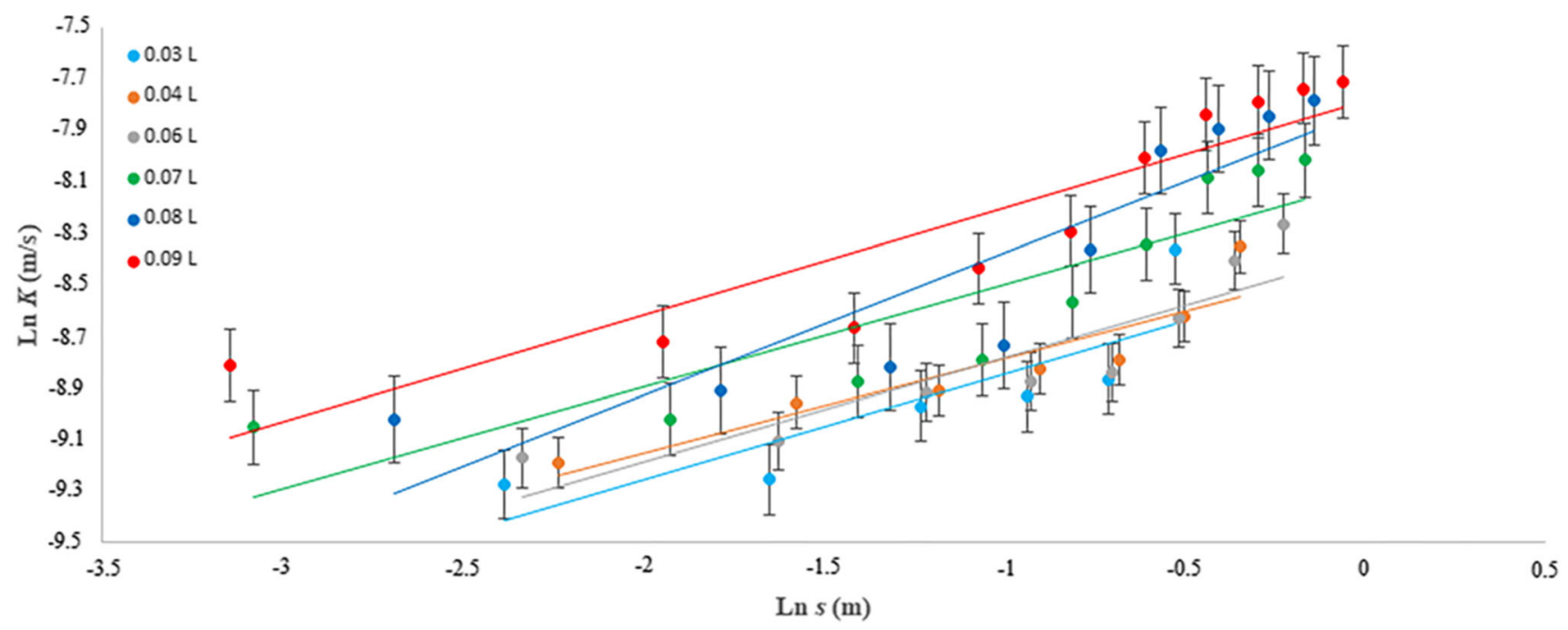

Fig. 2 Fitted scaling laws of $K$ data sets and corresponding values of $s$, relative to each injection volume

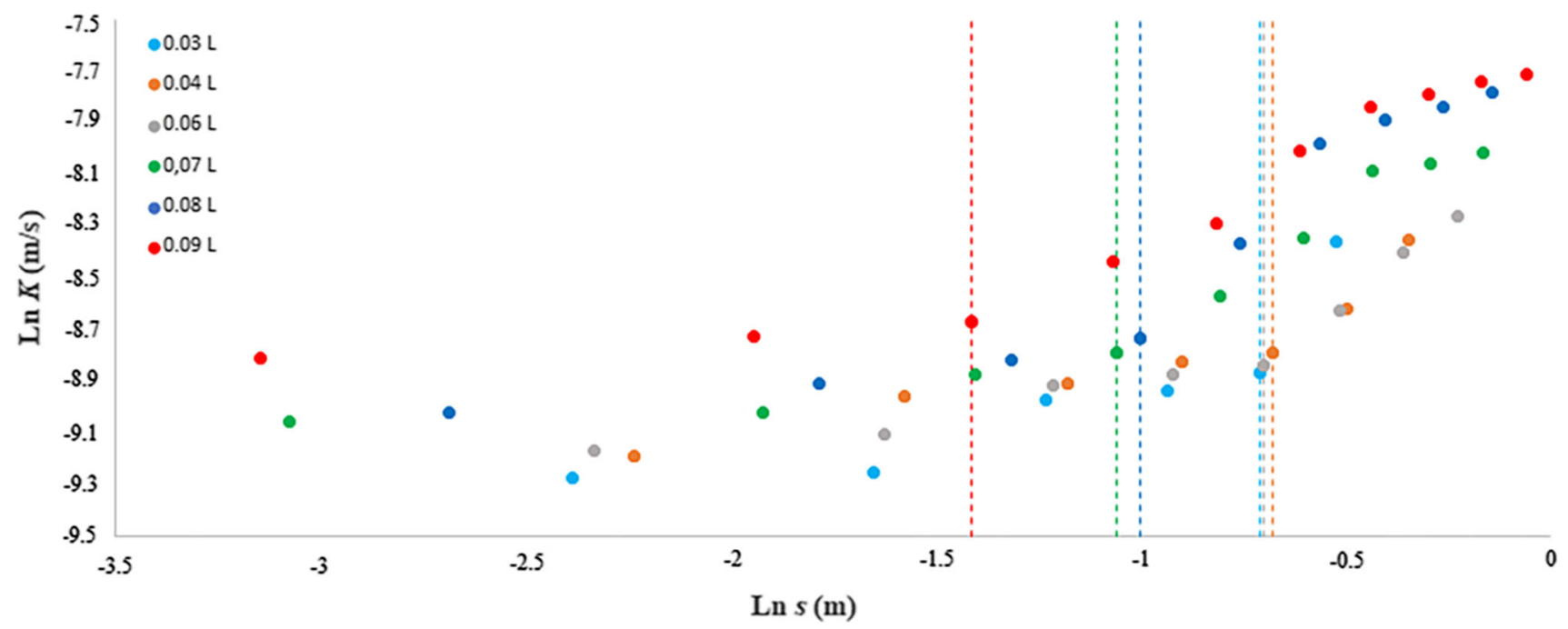

Fig. 3 Possible limit scale values of trend change, for the data sets $(K$, scale $)$ related to the injection volumes considered 
Table 5 Lower and upper cut-off limits for I and II ranges determined for the scaling behavior of $K$ in the data sets considered

\begin{tabular}{llllll}
\hline$V(\mathrm{~L})$ & I range & & & \multicolumn{2}{c}{ II range } \\
\cline { 2 - 3 } & $s_{I, \text { low }}$ & $s_{\text {I, upp }}$ & & $s_{\text {II low }}$ & $s_{\text {II }, \text { upp }}$ \\
\hline 0.03 & 0.092 & 0.492 & 0.492 & 0.592 \\
0.04 & 0.107 & 0.507 & 0.507 & 0.707 \\
0.06 & 0.097 & 0.497 & 0.497 & 0.797 \\
0.07 & 0.046 & 0.346 & 0.346 & 0.846 \\
0.08 & 0.068 & 0.368 & 0.368 & 0.868 \\
0.09 & 0.043 & 0.243 & 0.243 & 0.943 \\
\hline
\end{tabular}

Table 6 Values of the parameters $a$ and $b$ of the representative scaling laws of the $K$ scaling behavior relative to the I and II range and corresponding values of the coefficient $\mathrm{R}^{2}$, for the six data sets considered

\begin{tabular}{|c|c|c|c|c|c|c|}
\hline \multirow[t]{2}{*}{$V(\mathrm{~L})$} & \multicolumn{3}{|l|}{ I range } & \multicolumn{3}{|l|}{ II range } \\
\hline & $\begin{array}{l}a_{1} \\
\left(\mathrm{~m}^{(1-\mathrm{b})} / \mathrm{s}\right)\end{array}$ & $b_{1}$ & $\mathrm{R}^{2}$ & $\begin{array}{l}a_{2} \\
\left(\mathrm{~m}^{(1-\mathrm{b})} / \mathrm{s}\right)\end{array}$ & $b_{2}$ & $\mathrm{R}^{2}$ \\
\hline 0.03 & $1.68 \times 10^{-4}$ & 0.266 & 0.858 & $9.83 \times 10^{-4}$ & 2.738 & 1 \\
\hline 0.04 & $1.83 \times 10^{-4}$ & 0.252 & 0.970 & $3.63 \times 10^{-4}$ & 1.312 & 0.969 \\
\hline 0.06 & $1.70 \times 10^{-4}$ & 0.221 & 0.930 & $3.45 \times 10^{-4}$ & 1.243 & 0.997 \\
\hline 0.07 & $1.66 \times 10^{-4}$ & 0.125 & 0.803 & $4.15 \times 10^{-4}$ & 0.932 & 0.963 \\
\hline 0.08 & $1.86 \times 10^{-4}$ & 0.164 & 0.969 & $5.44 \times 10^{-4}$ & 1.117 & 0.918 \\
\hline 0.09 & $1.92 \times 10^{-4}$ & 0.081 & 0.994 & $5.04 \times 10^{-4}$ & 0.765 & 0.973 \\
\hline
\end{tabular}

Going beyond the two components model (5), Fig. 5 shows the scaling laws related to the I, II and III ranges, in which the investigation ranges of data sets, relating to injection volumes of $0.07 \mathrm{~L}, 0.08 \mathrm{~L}$ and $0.09 \mathrm{~L}$, were divided.

It is worth recalling that, by increasing the number of scaling ranges over two, there is not always a significant increase in $\mathrm{R}^{2}$. In the present case, for the injection volumes of $0.07 \mathrm{~L}, 0.08 \mathrm{~L}$ and $0.09 \mathrm{~L}$, the $\mathrm{R}^{2}$ values, for each of the three ranges into which the entire investigation range would be divided, are shown in Table 7 .

For the injection volume of $0.09 \mathrm{~L}$, the values of $\mathrm{R}^{2}$ obtained with three scaling ranges are completely comparable with those reported in Table 6 , obtained considering only two scaling ranges. For the other two injection volumes, i.e. $0.07 \mathrm{~L}$ and $0.08 \mathrm{~L}$, the values in Table 7 show that the increase is completely negligible than those of Table 6.

Returning to the case considered here with the subdivision of the investigation range into two parts, the change in $K$ scaling behavior (see Table 6, and Fig. 4) calls for a deeper analysis. In the present study it is clear that the investigation scale is not that characteristic of the laboratory, nor that of the field, but it is certainly an intermediate scale between these two. Therefore it is reasonable to assume that at smaller distances, for which the flow conditions are closer to those characteristics of the laboratory scale, the influence of heterogeneity is mainly exerted by the size and shape of the pores. While at large distances the influence of heterogeneity manifests itself mainly through the tortuosity of the porous medium. This explanation would lead to assign, for each injection volume considered, to the I range an influence of the heterogeneity on the $k$ scaling behavior, manifested mainly by the modalities of the laboratory scale, and to the II range by a predominant influence characteristic of the field scale. In addition, the impact of the injection volume is also important, and it has

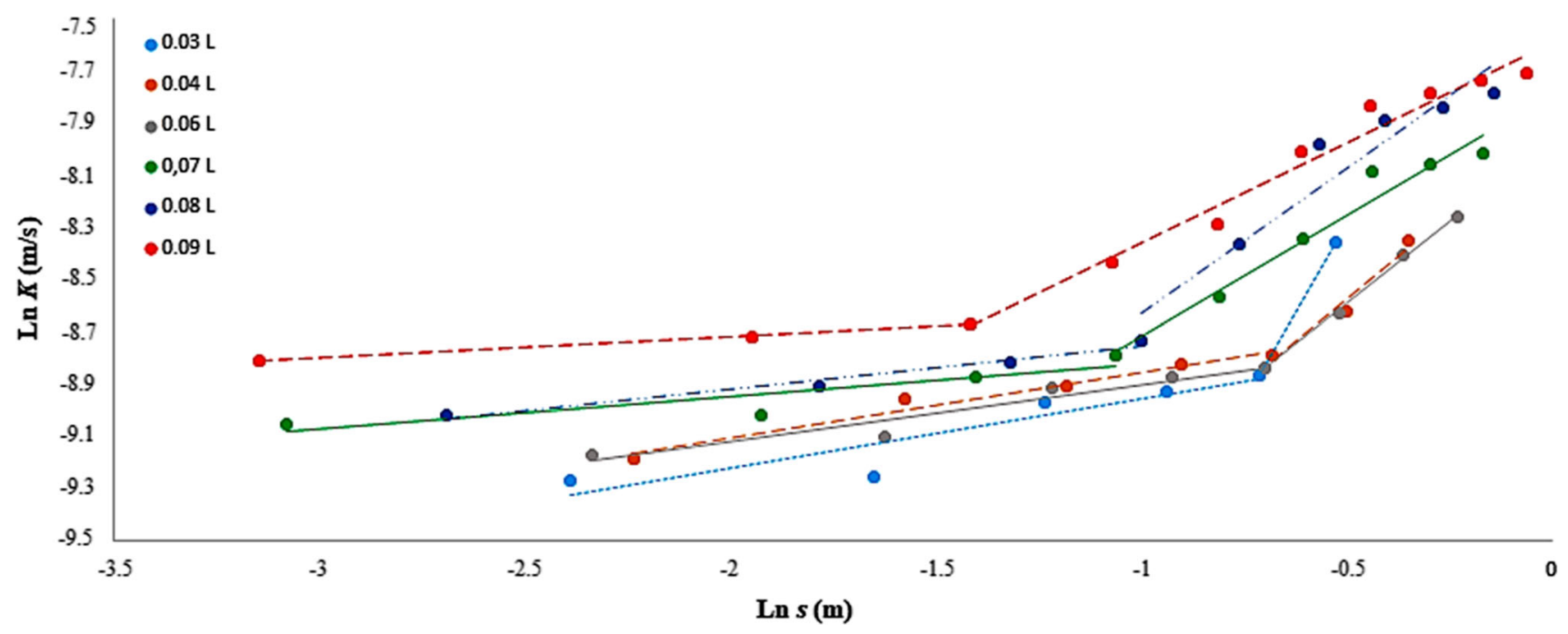

Fig. 4 Scaling laws related to the I and II ranges, for the six injection volumes considered 


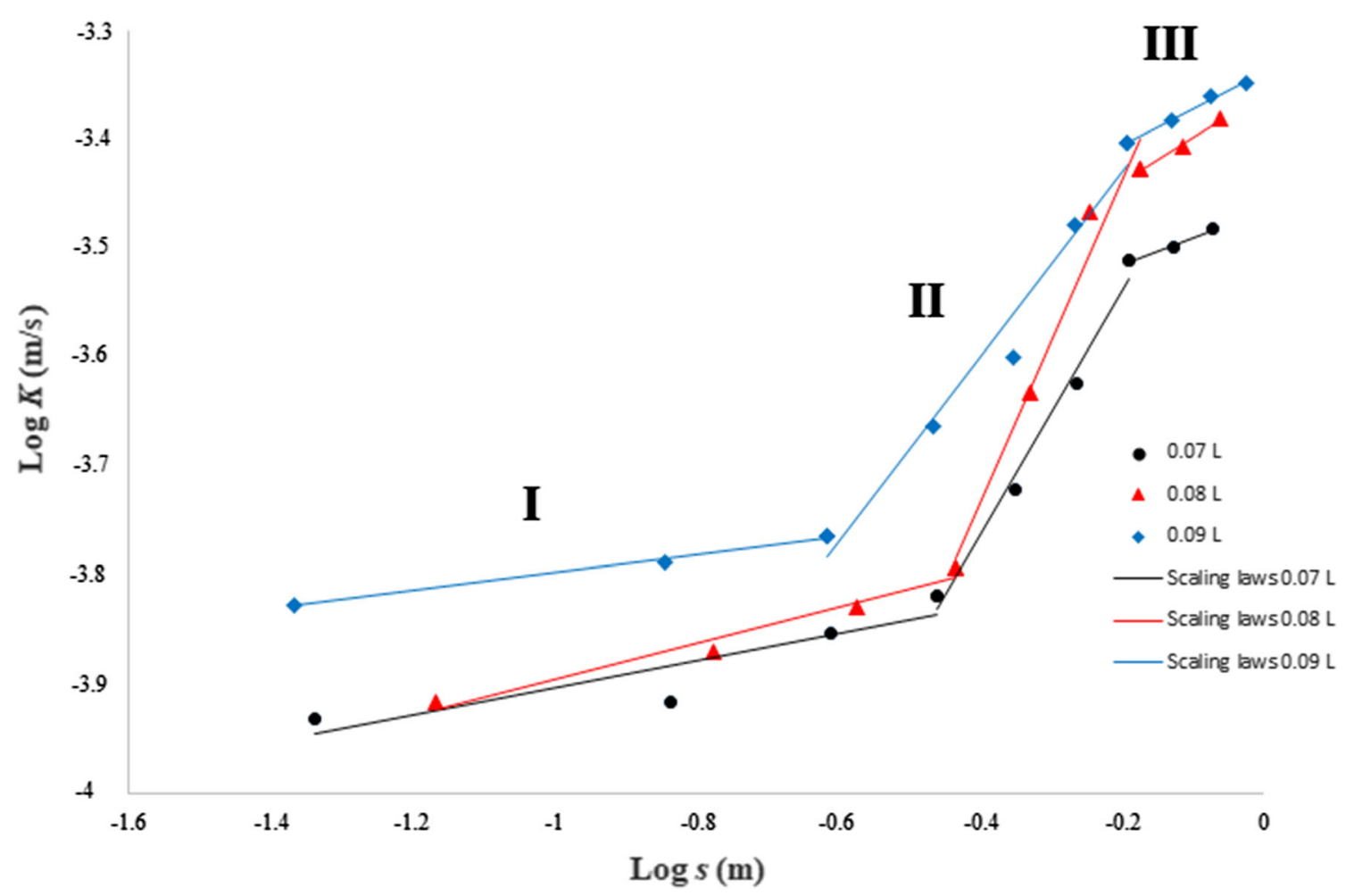

Fig. 5 Scaling laws related to the I, II and III ranges, for the injection volumes of $0.07 \mathrm{~L}, 0.08 \mathrm{~L}$ and $0.09 \mathrm{~L}$, assuming a division of the investigation range into three parts

Table $7 \mathrm{R}^{2}$ values for each range, assuming the division of the entire investigation range into three parts, for injection volumes of $0.07 \mathrm{~L}$, $0.08 \mathrm{~L}$ and $0.09 \mathrm{~L}$

\begin{tabular}{llll}
\hline Injection volumes & \multicolumn{2}{l}{$\mathrm{R}^{2}$ values } & \\
\cline { 2 - 4 } & I range & II range & III range \\
\hline 0.07 & 0.803 & 0.984 & 0.982 \\
0.08 & 0.969 & 0.995 & 0.993 \\
0.09 & 0.709 & 0.971 & 0.993 \\
\hline
\end{tabular}

to be accounted for. In fact, it is clear that for small injection volumes flow conditions are modest and closer to those characterizing the laboratory scale, while for the higher injection volumes the flow conditions are closer to those of the field scale. As a consequence, scaling was achieved by grouping results obtained at the lowest injection volumes, together with the intermediate and the largest ones. In this way, three groups of scaling laws were obtained. The group No. 1 consists of the scaling laws relating to the injection volumes of $0.03 \mathrm{~L}, 0.04 \mathrm{~L}$ and 0.06 L; group No. 2 consists of the scaling laws relating to the injection volumes of $0.07 \mathrm{~L}$ and $0.08 \mathrm{~L}$; finally, group No. 3 consists of the scaling laws related to the single injection volume equal to $0.09 \mathrm{~L}$. For each of these three groups, we identified a single scaling law representative of the I range and, in a similar way, another for the II range. The values of parameters $a$ and $b$ characterizing the scaling laws related to the I and the II range for the three groups defined above are shown in Table 8 .

Data of Table 8 show that, for the both I and II ranges, the values of $a$ tend to increase, ranging from the group No. 1 to the No. 3. The values of $a$ vary for the I range between $1.74 \times 10^{-4} \mathrm{~m}^{(1-\mathrm{b})} / \mathrm{s}$ and $1.92 \times 10^{-4} \mathrm{~m}^{(1-\mathrm{b})} / \mathrm{s}$, while for the II range they vary between $3.58 \times 10^{-4} \mathrm{~m}^{(1-b)} / \mathrm{s}$ and $5.04 \times 10^{-4} \mathrm{~m}^{(1-\mathrm{b})} / \mathrm{s}$. The values of $b$ are also very close, varying for the I range between 0.081 and 0.250 , while for the II range they vary between 0.765 and 1.250 . Figure 6 shows the scaling laws, relating to the I and II ranges, of the Group No. 1, that is for the injection volumes of $0.03 \mathrm{~L}$, $0.04 \mathrm{~L}$ and $0.06 \mathrm{~L}$.

In the Fig. 6 it is also seen the interval of confidence (both at $95 \%$ and $99 \%$ ) of the Group I. The scaling laws have a much more regular trend in the I range than in the II range. Moreover, all the scaling laws of the I range are included within the $95 \%$ and $99 \%$ confidence intervals. In the II range the scaling laws relating to the injection volumes of $0.04 \mathrm{~L}$ and $0.06 \mathrm{~L}$ are regular and very close to the overall scaling law, while the scaling law relative to the injection volume of $0.03 \mathrm{~L}$ is meaningless, since it was derived for only two points. Similarly, Fig. 7 shows the 
Table 8 Values of the parameters $a$ and $b$ defining the scaling law (5), related to the I and II ranges, for groups of scaling laws No. 1 , No. 2 and No. 3, namely for increasing injection volumes, and related values of $\mathrm{R}^{2}$

\begin{tabular}{llllllll}
\hline Groups & I Range & \multicolumn{7}{l}{ II Range } \\
\cline { 2 - 3 } & $\begin{array}{l}a_{1} \\
\left(\mathrm{~m}^{(1-\mathrm{b})} / \mathrm{s}\right)\end{array}$ & $b_{1}$ & $\mathrm{R}^{2}$ & & $\begin{array}{l}a_{2} \\
\left(\mathrm{~m}^{(1-\mathrm{b})} / \mathrm{s}\right)\end{array}$ & $b_{2}$ & $\mathrm{R}^{2}$ \\
\hline Group No. 1 & $1.74 \times 10^{-4}$ & 0.250 & 0.834 & & $3.58 \times 10^{-4}$ & 1.250 & 0.849 \\
Group No. 2 & $1.76 \times 10^{-4}$ & 0.144 & 0.848 & & $4.80 \times 10^{-4}$ & 1.040 & 0.871 \\
Group No. 3 & $1.92 \times 10^{-4}$ & 0.081 & 0.994 & & $5.04 \times 10^{-4}$ & 0.765 & 0.973 \\
\hline
\end{tabular}

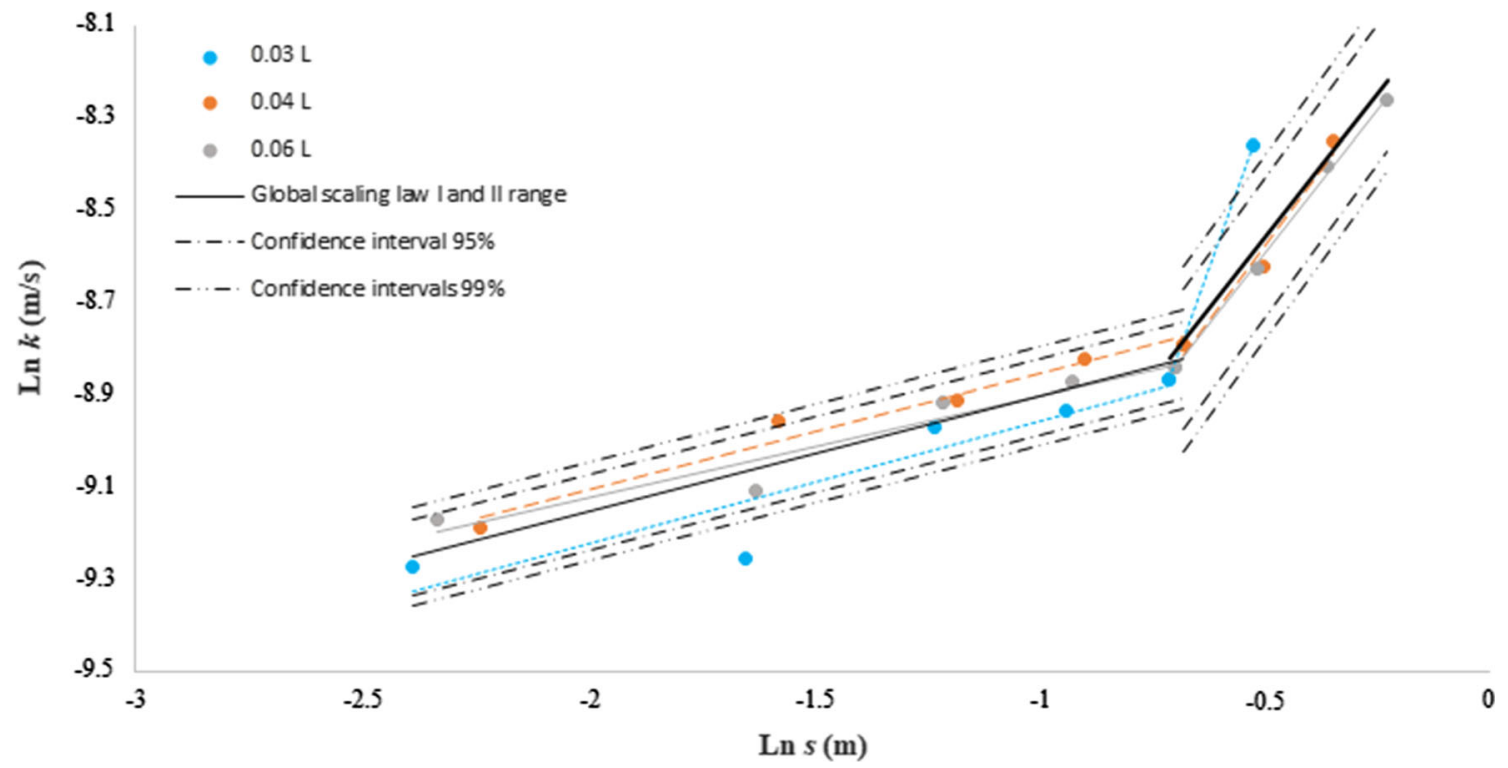

Fig. 6 Scaling laws, relating to the I and II ranges, of the Group No. 1, with global scaling law and 95\% and 99\% confidence intervals

scaling laws, relating to the I and II ranges, of the Group No. 2, that is for the injection volumes of $0.07 \mathrm{~L}$ and 0.08 L.

Figure 7 shows that the scaling laws relating to the individual injection volumes falling within Group No. 2, that is $0.07 \mathrm{~L}$ and $0.08 \mathrm{~L}$, show a very regular trend both in the I range and in the II range, largely within the confidence intervals taken into consideration. Figure 8 shows, in relation to the I and II ranges, the only scaling law of the

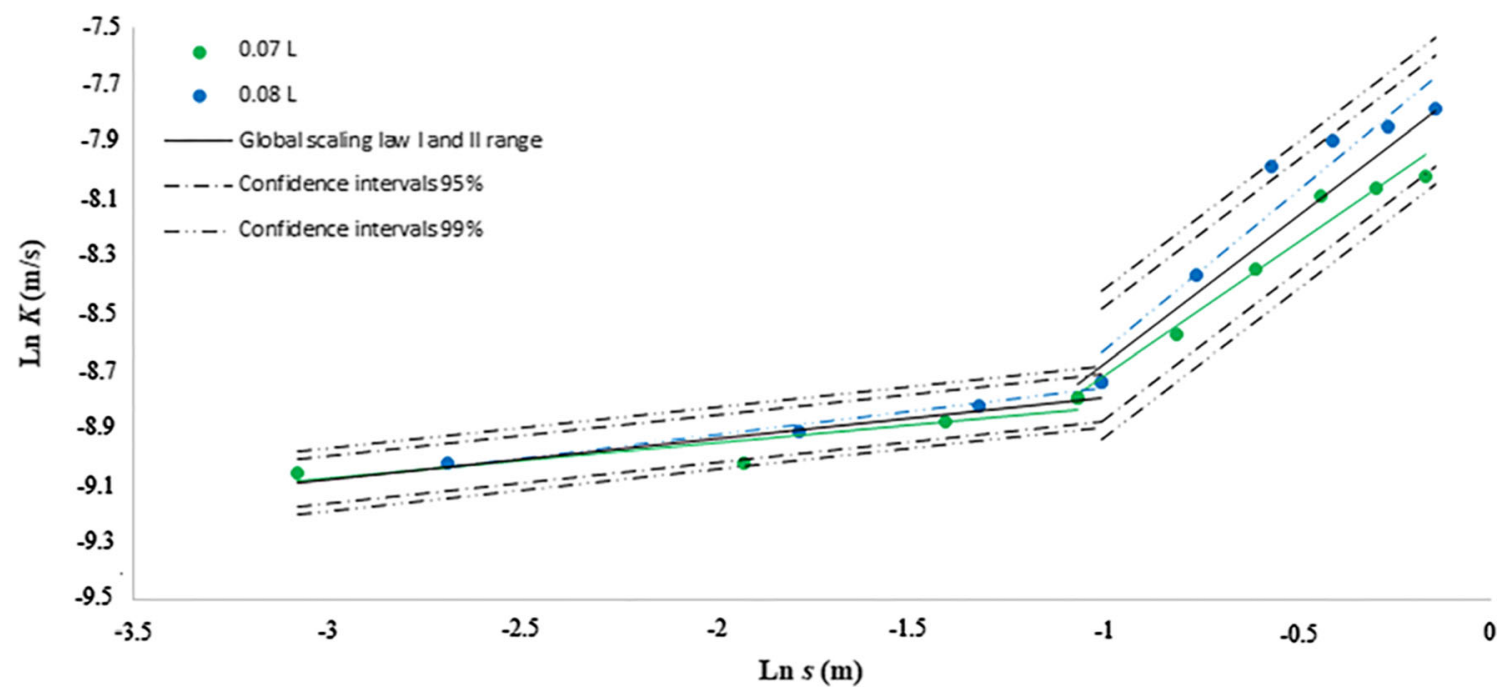

Fig. 7 Scaling laws, relating to the I and II ranges, of Group No. 2, with global scaling law and 95\% and 99\% confidence intervals 
Group No. 3, that is the one relating to the injection volume of $0.09 \mathrm{~L}$, already shown in the graph of Fig. 4.

Figure 9 shows, in relation to both I and II ranges, the global scaling laws representative of the Groups No. 1, No. 2 and No. 3. Figure 9 shows that passing from the Group No. 1 to the Group No. 3 the values of $k$ tend to increase, for both ranges.

This shows that the injection volume can also be usefully taken as a scaling parameter. Moreover, the same graph shows that the increase of $K$ with the scale is much faster in the II range compared to I. This can be attributed to the different ways in which heterogeneity exerts its influence in the I and II ranges. However, it should also be noted that the increase in the volume introduced during the slug test regards an increase in the aquifer volume involved in the measurement, namely the increase of the radius of influence. This is also remarked by the fact that the amplitude of the II range tends to increase passing from Group I to the following ones. Figure 9 also shows that the amplitude of the I range tends to be reduced, going from the I Group to the following ones. This could be addressed to the fact that as the injection volume increases, the influence of the heterogeneity tends to manifest itself mainly with the characteristic modalities of the field scale. Therefore, it could also be hypothesized that with increasing injection volumes it is possible to switch from the modalities characteristic of laboratory to those of the field. On this basis it could be stated that for data sets obtained with low injection volumes, heterogeneity manifests itself mainly in the specific modalities of the laboratory scale. As larger injection volumes are considered, scale values are reached for which heterogeneity manifests itself at first with both the modalities of the laboratory and field scale and then mainly with those of the field scale.

\section{Conclusions}

The description of the scaling behavior of the aquifers' parameters, in particular of the hydraulic conductivity, requires carrying out a careful analysis of the experimental data, which allows to identify the most suitable scaling law. The most used mathematical model is the power type (3). If this law is valid in the whole range of scale, the approach to be used is that of the simple-scaling type. In many cases, to obtain a more reliable description, it is convenient to use a multi-scaling approach. This "piecewise approach" allows a better description of the scaling behavior of the investigated parameter, which in the entire range examined does not present a single trend, but assumes trends that can be represented with different scaling laws.

The choice between a simple-scaling or multi-scaling approach must always be made on the basis of the values assumed by the coefficient of determination $\mathrm{R}^{2}$. This can be done by comparing the value that $\mathrm{R}^{2}$ assumes using a unique scaling law, relating to the simple-scaling approach, with those that this parameter assumes using the scaling laws obtained from a possible multi-scaling approach. In the present study it is shown that the choice between the use of a simple-scaling or multi-scaling approach can be greatly facilitated by a simple graphical control of the trend presented by the experimental points. More precisely, the arrangement of the experimental points in the graph of Fig. 2 clearly shows the presence of two different $K$ trends as the distance $s$ varies. Hence, the opportunity to use a

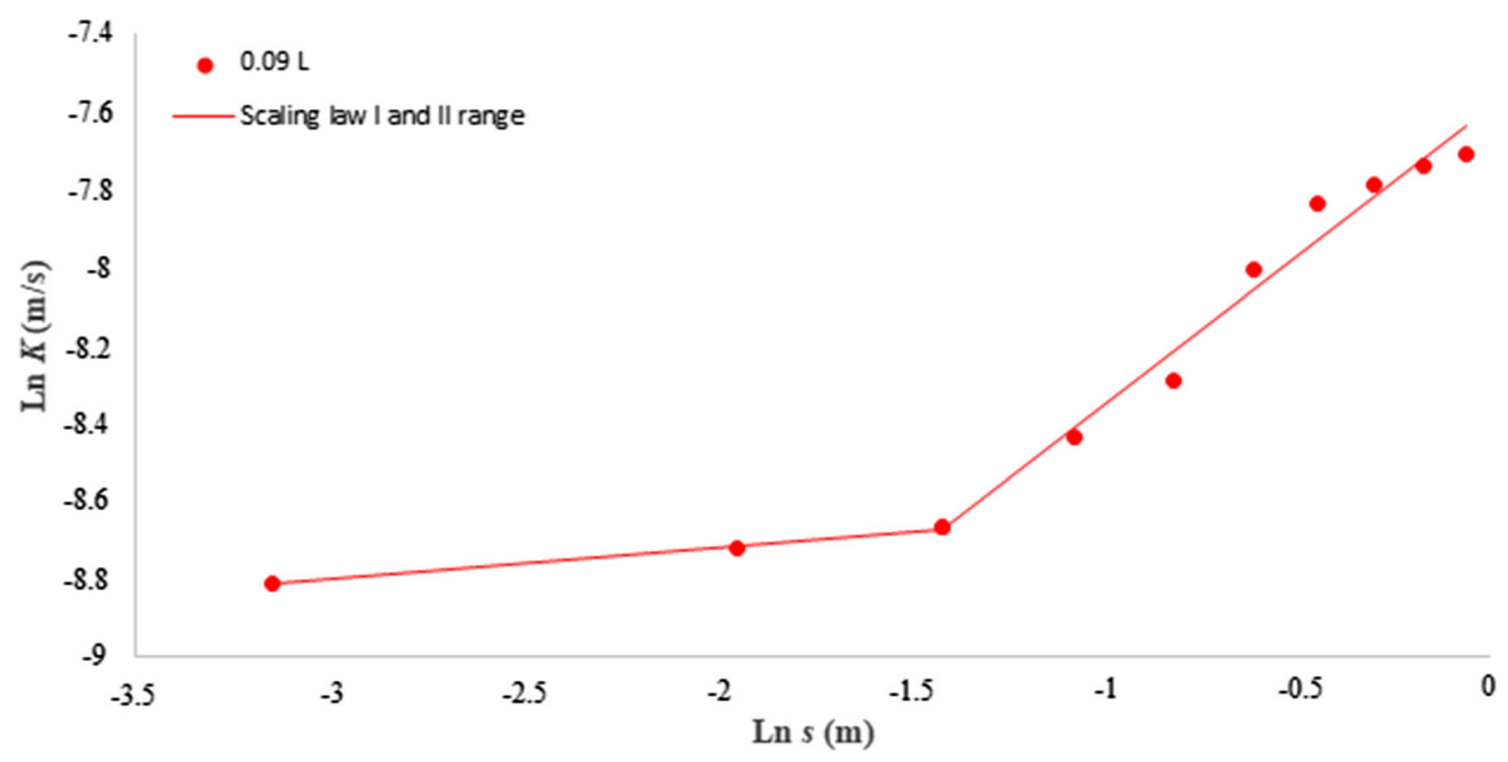

Fig. 8 Scaling law, relating to the I and II ranges, of the Group No. 3 


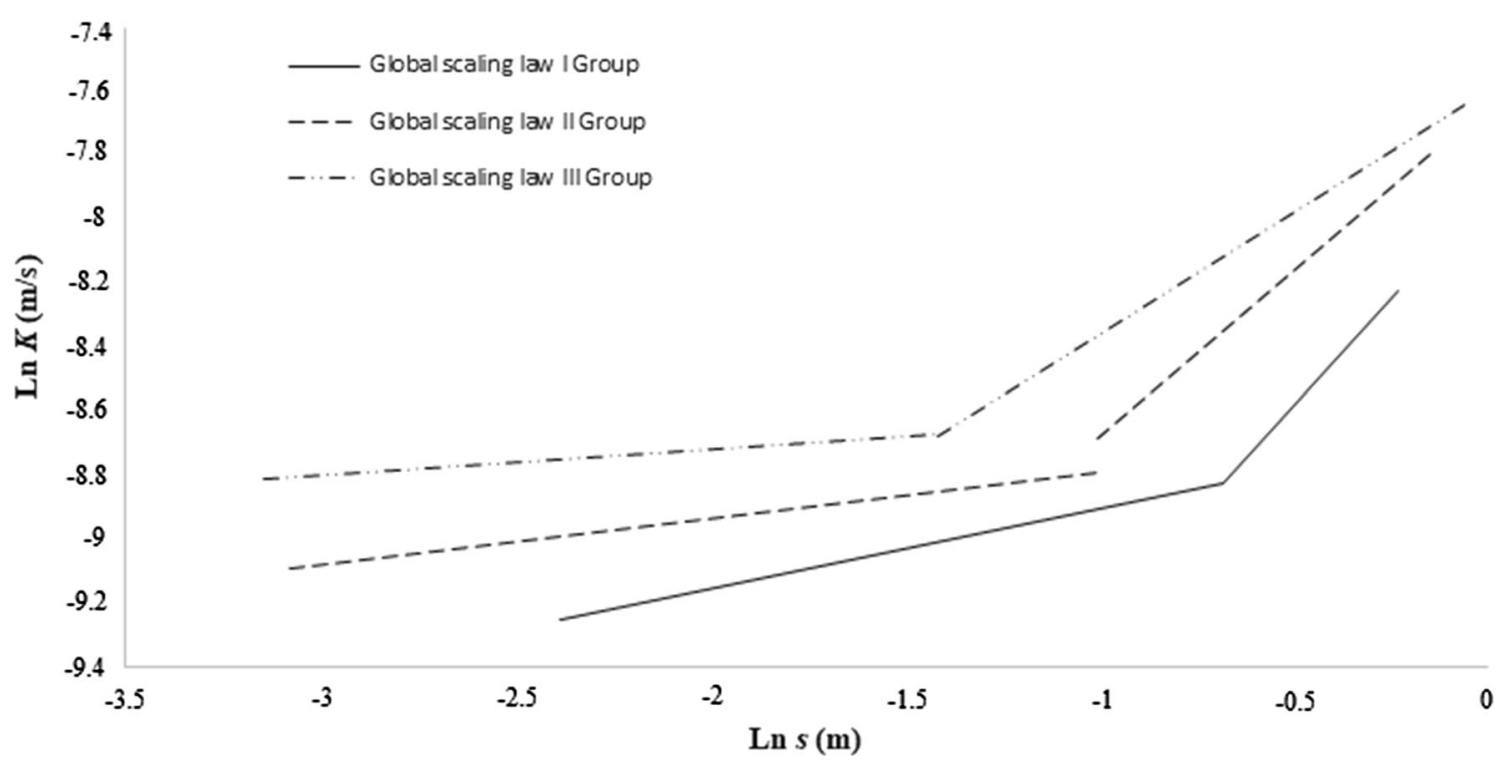

Fig. 9 Global scaling laws of Groups No. 1, No. 2 and No. 3, for the I and II ranges

multi-scaling approach is evident, by using Eq. (5). This is also confirmed by the comparison between the $\mathrm{R}^{2}$ values shown in Table 4. Then, the values of the cut-off limits, which identify the bounds of each range wherein the whole range of investigation is divided, have been determined. Moreover, the mesoscale, which is intermediate between the laboratory one and that characteristic of the field, allowed to detect the variation of the ways in which the heterogeneity exerts its influence on the $K$ scaling behavior, under flow conditions induced by different injection volumes.

One of the most important results is that the value of the injection volume has great influence. In fact, the present study has shown that the small injection volumes are generally related to higher values of the wideness of the I range, and therefore the ways in which the influence of heterogeneity is manifested are mainly those of the laboratory scale. With increasing injection volume, the width of the II range increases, and concurrently the ways in which the influence of heterogeneity manifests itself get closer and closer to those of field (Fig. 8).

As a consequence, moving from group I to group III, the modalities through which the influence of heterogeneity is exerted tend to move away from those mainly typical of the laboratory scale and to get closer and closer to those of the field scale. In this context, the situation of the intermediate scale, in which the influence of heterogeneity manifests itself simultaneously through the laboratory and field modalities, assumes great importance. Results of a doublescaling analysis were also presented in this study. Of course, straightforward extension to higher scaling is possible, and the present manuscript represents a first step toward it. The topics taken into consideration in the present study, relative to a scale intermediate between the laboratory and the field, are numerous and interesting, such as the deepening of the knowledge of the aspects related to the heterogeneity influence and the attempts to give greater continuity to the representation of the scaling behavior of the aquifer characteristic parameters (Severino et al. 2008; Severino 2019). Some aspects were investigated (Chevalier et al. 2001; Fallico et al. 2018) and many others are part of ongoing projects.

Acknowledgements The present manuscript has no funding to acknowledge.

Funding Open access funding provided by Università degli Studi di Napoli Federico II within the CRUI-CARE Agreement.

\section{Declarations}

Conflict of interest The authors declare that they have no conflict of interest.

Open Access This article is licensed under a Creative Commons Attribution 4.0 International License, which permits use, sharing, adaptation, distribution and reproduction in any medium or format, as long as you give appropriate credit to the original author(s) and the source, provide a link to the Creative Commons licence, and indicate if changes were made. The images or other third party material in this article are included in the article's Creative Commons licence, unless indicated otherwise in a credit line to the material. If material is not included in the article's Creative Commons licence and your intended use is not permitted by statutory regulation or exceeds the permitted use, you will need to obtain permission directly from the copyright holder. To view a copy of this licence, visit http://creativecommons. org/licenses/by/4.0/. 


\section{References}

Aristodemo F, Ianchello M, Fallico C (2018) Smoothing analysis of slug tests data for aquifer characterization at laboratory scale. J Hydrol 562:125-139

Bird NRA, Perrier E (2010) Multiscale percolation properties of a fractal pore network. Geoderma 160(1):105-110

Bouma J (1982) Measuring the hydraulic conductivity of soil horizons with continuous macropores. Soil Sci Soc Am J 46(2):438-441

Broyda S, Dentz M, Tartakovsky DM (2010) Probability density functions for advective-reactive transport in radial flow. Stoch Environ Res Risk Assess 24:985-992. https://doi.org/10.1007/ s00477-010-0401-4

Butler JJ Jr (1997) The design, performance, and analysis of slug tests. Lewis Publishers, Boca Raton

Chevalier S, Bués MA, Tournebize J, Banton O (2001) Stochastic delineation of wellhead protection area in fractured aquifers and parametric sensitivity study. Stoch Environ Res Risk Assess 15:205-227. https://doi.org/10.1007/PL00009790

Dagan G (1989) Flow and transport in porous formation. Springer, New York

De Bartolo S, Fallico C, Veltri M (2013) A note on the fractal behavior of hydraulic conductivity and effective porosity for experimental values in a confined aquifer. Sci World J. https:// doi.org/10.1155/2013/356753

Di Federico V, Neuman SP (1998) Flow in multiscale log conductivity fields with truncated power variograms. Water Resour Res 34(5):975-987. https://doi.org/10.1029/98WR00220

Di Federico V, Neuman SP, Tartakovsky DM (1999) Anisotropy, lacunarity, and upscaled conductivity and its autocovariance in multiscale random fields with truncated power variograms. Water Resour Res 35(10):2891-2908. https://doi.org/10.1029/ 1999WR900158

Fallico C (2014) Reconsideration at field scale of the relationship between hydraulic conductivity and porosity. The case of a sandy aquifer in South Italy. Sci World J. https://doi.org/10. $1155 / 2014 / 537387$

Fallico C, De Bartolo S, Troisi S, Veltri M (2010) Scaling analysis of hydraulic conductivity and porosity on a sandy medium of an unconfined aquifer reproduced in the laboratory. Geoderma 160(1):3-12

Fallico C, Vita MC, De Bartolo S, Straface S (2012) Scaling effect of the hydraulic conductivity in a confined aquifer. Soil Sci 177(6):385-391

Fallico C, De Bartolo S, Veltri M, Severino G (2016) On the dependence of the saturated hydraulic conductivity upon the effective porosity through a power law model at different scales. Hydrol Process. https://doi.org/10.1002/hyp.10798

Fallico C, Ianchello M, De Bartolo S, Severino G (2018) Spatial dependence of the hydraulic conductivity in a well-type configuration at the mesoscale. Hydrol Process 32(4):590-595. https:// doi.org/10.1002/hyp. 11422

Fallico C, De Bartolo S, Brunetti G, Severino G (2020) Use of fractal models to define the scaling behavior of the aquifers' parameters at the mesoscale. Stoch Environ Res Risk Assess. https://doi.org/ 10.1007/s00477-020-01881-2

Giménez D, Rawls WJ, Lauren JG (1999) Scaling properties of saturated hydraulic conductivity in soil. Geoderma 88(3-4):205-220

Harp DR, Vesselinov VV (2010) Stochastic inverse method for estimation of geostatistical representation of hydrogeologic stratigraphy using borehole logs and pressure observations.
Stoch Environ Res Risk Assess 24:1023-1042. https://doi.org/ 10.1007/s00477-010-0403-2

Hunt AG (2006) Scale-dependent hydraulic conductivity in anisotropic media from dimensional cross-over. Hydrogeol $\mathbf{J}$ 14:499-507

Hvorslev MJ (1951) Time lag and soil permeability in ground-water observations. Bull. N. 36, Waterways Exper. Sta. Corps of Engrs, U.S. Army, Vicksburg, Mississippi, pp 1-50

Indelman P (2004) On macrodispersion in uniform-radial divergent flow through weakly heterogeneous aquifers. Stoch Environ Res Risk Assess 18:16-21. https://doi.org/10.1007/s00477-0030165-1

Knudby C, Carrera J (2006) On the use of apparent hydraulic diffusivity as an indicator of connectivity. J Hydrol 329(3-4):377-389

Neuman SP, Di Federico V (2003) Multifaceted nature of hydrogeologic scaling and its interpretation. Rev Geophys 41(3):1014. https://doi.org/10.1029/2003RG000130

Sánchez-Vila X, Carrera J, Girardi JP (1996) Scale effects in transmissivity. J Hydrol 183(1-2):1-22. https://doi.org/10.1016/ S0022-1694(96)80031-X

Schulze-Makuch D, Cherkauer DS (1998) Variations in hydraulic conductivity with scale of measurement during aquifer tests in heterogeneous, porous carbonate rocks. Hydrogeol J 6(2):204-215

Severino G (2011a) Stochastic analysis of well-type flows in randomly heterogeneous porous formations. Water Resour Res 47:W03520. https://doi.org/10.1029/2010WR009840

Severino G (2011b) Macrodispersion by point-like source flows in randomly heterogeneous porous media. Transp Porous Media 89:121-134. https://doi.org/10.1007/s11242-011-9758-1

Severino G (2019) Effective conductivity in steady well-type flows through porous formations. Stoch Environ Res Risk Assess 33(3):827-835. https://doi.org/10.1007/s00477-018-1639-5

Severino G, Santini A (2005) On the effective hydraulic conductivity in mean vertical unsaturated steady flows. Adv Water Resour 28:964-974. https://doi.org/10.1016/j.advwatres.2005.03.003

Severino G, Coppola A (2012) A note on the apparent conductivity of stratified porous media in unsaturated steady flow above a water table. Transp Porous Media 91(2):733-740. https://doi.org/10. 1007/s11242-011-9870-2

Severino G, De Bartolo S (2015) Stochastic analysis of steady seepage underneath a water-retaining wall through highly anisotropic porous media. J Fluid Mech 778:253-272

Severino G, Santini A, Sommella A (2008) Steady flows driven by sources of random strength in heterogeneous aquifers with application to partially-penetrating wells. Stoch Environ Res Risk Assess 22:567-582. https://doi.org/10.1007/s00477-0070175-5

Severino G, Santini A, Monetti VM (2009) Modelling water flow and solute transport in heterogeneous unsaturated porous media. In: Pardalos and Papajorgji (eds) Advances in modelling agricultural systems, pp 361-383. https://doi.org/10.1007/978-0-387-75181817

Severino G, Comegna A, Coppola A, Sommella A, Santini A (2010) Stochastic analysis of a field-scale unsaturated transport experiment. Adv Water Resour 33:1188-1198

Severino G, Santini A, Sommella A (2011) Macrodispersion by diverging radial flows in randomly heterogeneous porous media. J Contam Hydrol 123:40-49. https://doi.org/10.1016/j.jconhyd. 2010.12.005

Severino G, De Bartolo S, Brunetti G, Sommella A, Fallico C (2019) Experimental evidence of the stochastic behavior of the 
conductivity in radial flow configurations. Stoch Environ Res Risk Assess 33(8):1651-1657. https://doi.org/10.1007/s00477019-01704-z

Yanuka M, Dullien FAL, Elrick DE (1986) Percolation processes and porous media. I. Geometrical and topological model of porous media using a three-dimensional joint pore size distribution. J Colloid Interface Sci 112(1):24-41

Publisher's Note Springer Nature remains neutral with regard to jurisdictional claims in published maps and institutional affiliations. 